\title{
Efeitos da campanha nacional de prevenção ao câncer da pele, em Alfenas-MG, no
}

\section{período de 2014 a 2018}

\author{
Effects of the national skin cancer prevention campaign, in Alfenas-MG, during the period from 2014 to
} 2018

Efectos de la campaña nacional de prevención del cáncer de piel en Alfenas-MG durante el período de 2014 a 2018

Letícia Natsumi Koga

ORCID: https://orcid.org/0000-0002-0763-9611 Universidade Federal de Alfenas, Brasil

E-mail: leticia.koga@ sou.unifal-mg.edu.br

Éllen Maria de Almeida

ORCID: https://orcid.org/0000-0003-0474-1327 Universidade Federal de Alfenas, Brasil

E-mail:ellen.almeida@sou.unifal-mg.edu.br

Cristiane Gomes Fernandes Freire ORCID: https://orcid.org/0000-0002-2490-0690 Universidade Federal de Alfenas, Brasil

E-mail: cristiane.freire@ sou.unifal-mg.edu.br José Jerônimo Souza Freire

ORCID: https://orcid.org/0000-0003-4717-3067 Universidade Federal de Alfenas, Brasil E-mail: jose.freire@ sou.unifal-mg.edu.br

Carla Cristina dos Santos Campos

ORCID: https://orcid.org/0000-0001-5960-7323 Universidade Federal de Alfenas, Brasil E-mail:carla.campos@unifal-mg.edu.br

\begin{abstract}
Resumo
Introdução: O câncer de pele é uma das doenças malignas mais comuns no mundo. O número de casos novos de câncer de pele não melanoma esperados, para cada ano do triênio 2020-2022, é de 83.770 em homens e de 93.160 em mulheres e, em relação ao câncer de pele melanoma, o número de casos novos estimados é de 4.200 em homens e de 4.250 em mulheres. São vários os fatores de risco para a doença, destacando-se a exposição solar sem proteção como fator principal. Objetivos: Avaliar os efeitos da Campanha Nacional de Prevenção ao Câncer de Pele (CNPCP), na cidade de Alfenas-Minas Gerais, nos anos de 2014 a 2018. Métodos: Pesquisa documental sobre os relatórios das campanhas do Dia Nacional de Combate ao Câncer da Pele, entre os anos de 2014 e 2018, disponibilizados pela Sociedade Brasileira de Dermatologia. Resultados: Foram realizados um total de 1291 exames dermatológicos, sendo diagnosticados 108 casos de câncer de pele. Entre os examinados, cerca de $58,4 \%$ se expunham ao sol sem proteção. As mulheres eram a maioria nos exames, representando uma porcentagem de, aproximadamente, $68,8 \%$. O número de pessoas com risco de câncer de pele manteve-se maior durante todos os anos analisados, totalizando 756 indivíduos. Considerações finais: A CNPCP foi importante para examinar e diagnosticar pacientes durante o período analisado na cidade de Alfenas, mas seus dados também evidenciaram que os cuidados com a exposição solar pela população ainda precisam melhorar, sobretudo entre o público masculino, o qual participou menos das campanhas já realizadas.
\end{abstract}

Palavras-chave: Câncer da pele; Melanoma; Carcinoma basocelular; Carcinoma de células escamosas; Radiação solar.

\begin{abstract}
Introduction: Skin cancer is one of the most common malignancies in the world. The expected number of non-melanoma skin cancer new cases, for each year of the 2020-2022 triennium, is 83,770 in men and 93,160 in women and, concerning melanoma skin cancer, the estimated number of new cases is 4,200 in men and 4,250 in women. There are several risk factors for the disease, highlighting unprotected sun exposure as the main factor. Objectives: To evaluate the effects of the National Skin Cancer Prevention Campaign (CNPCP), in the city of Alfenas-Minas Gerais, in the years 2014 to 2018. Methods: Documentary research on the National Skin Cancer Fight Day campaigns reports, between 2014 and 2018, made available by the Brazilian Society of Dermatology. Results: A total of 1291 dermatological examinations were performed, and 108 cases of skin cancer
\end{abstract}


were diagnosed. Among those examined, about 58.4\% exposed themselves to the sun without protection. Women were the majority in the exams, representing a percentage of approximately $68.8 \%$. The number of people at risk for skin cancer remained higher during all the years analyzed, totaling 756 individuals. Final considerations: The CNPCP was important for examining and diagnosing patients in the city of Alfenas during the analyzed period, but its data also showed that the sun exposure care by the population still needs to improve, especially among the male public, who participated less in the analyzed campaigns.

Keywords: Cancer of the skin; Melanoma; Carcinoma, Basal cell; Carcinoma, Squamous cell; Solar radiation.

\section{Resumen}

Introducción: el cáncer de piel es una de las neoplasias malignas más frecuentes en el mundo. El número de nuevos casos de cáncer de piel no melanoma previstos, para cada año del trienio 2020-2022, es de 83.770 en hombres y 93.160 en mujeres y, en relación con el cáncer de piel melanoma, el número estimado de nuevos casos es de 4.200 en hombres y 4.250 en mujeres. Existen varios factores de riesgo para la enfermedad, destacando la exposición al sol sin protección como el factor principal. Objetivos: Evaluar los efectos de la Campaña Nacional de Prevención del Cáncer de Piel (CNPCP), en la ciudad de AlfenasMinas Gerais, entre 2014 y 2018. Métodos: Investigación documental sobre los informes de las campañas del Día Nacional de Lucha contra el Cáncer de Piel, entre 2014 y 2018, puesto a disposición por la Sociedad Brasileña de Dermatología. Resultados: Se realizaron un total de 1291 exámenes dermatológicos y se diagnosticaron 108 casos de cáncer de piel. Entre los examinados, alrededor del $58,4 \%$ se expusieron al sol sin protección. Las mujeres fueron mayoría en los exámenes, representando un porcentaje de aproximadamente $68,8 \%$. El número de personas en riesgo de cáncer de piel se mantuvo mayor durante todos los años analizados, totalizando 756 individuos. Consideraciones finales: El CNPCP fue importante para el examen y diagnóstico de los pacientes durante el período analizado en la ciudad de Alfenas, pero sus datos también mostraron que la atención a la exposición solar por parte de la población aún debe mejorar, especialmente entre el público masculino, que participó menos en campañas ya realizadas.

Palabras clave: Cáncer de la piel; Melanoma; Carcinoma basocelular; Carcinoma de células escamosas; Radiación solar.

\section{Introdução}

O câncer de pele é uma das doenças malignas mais comuns no mundo, com aproximadamente 350.000 melanomas e 13 milhões de cânceres de pele não melanoma diagnosticados todo ano, resultando em torno de 81.000 mortes (Silva et al., 2018). No Brasil, o número estimado de novos casos de câncer de pele não melanoma, em 2020, foi de 176.930 e o número de novos casos do subtipo de melanoma foi de 8.450. Além disso, o número de casos novos de câncer de pele não melanoma esperados, para cada ano do triênio 2020-2022, é de 83.770 em homens e de 93.160 em mulheres e, em relação ao câncer de pele melanoma, o número de casos novos estimados é de 4.200 em homens e de 4.250 em mulheres (INCA, 2019). Os três tipos mais frequentes de câncer de pele são carcinoma basocelular (CBC), carcinoma espinocelular (CEC) e melanoma, sendo este o mais letal (Leiter, Keim, \& Garbe, 2020).

O CBC é responsável por $75 \%$ de todos os cânceres de pele e é o tumor maligno mais comum em populações brancas (Peris et al., 2019). É subclassificado em nodular, que se apresenta por meio de pápulas císticas nacaradas cor de rosa ou brancas ou nódulos que têm telangiectasia na sua superfície e podem ser ulceradas; superficial, com placas eritematosas, bem demarcadas, escamosas e bordas branco-peroladas; esclerosante ou infiltrativo, caracterizando-se como lesão cerosa, da cor da pele, semelhante a cicatriz, tende a recorrer e pode infiltrar os nervos cutâneos; pigmentado, que surge como lesão marrom, azul ou acinzentada; e basoescamoso, o qual tem características mistas de CBC e CEC (Jones et. al, 2020).

Os CECs manifestam-se como pápulas, placas ou nódulos eritematosos e ceratóticos que surgem por lesão actínica e podem apresentar ulceração, sangramento intermitente e ausência de cicatrização (Brandt \& Moore, 2019). Já o melanoma pode ser classificado em superficial, que se apresenta como uma lesão cutânea pigmentada nova ou que mudou de tamanho, forma ou cor; nodular, em forma de pápula ou nódulo firme, simétrico e uniformemente pigmentado, que pode ulcerar e sangrar; lentigo maligno, que desenvolve-se como uma mácula pigmentada precursora de crescimento lento; e lentiginoso acral, o qual ocorre exclusivamente nas palmas das mãos e plantas dos pés e sob as unhas, não estão relacionados à exposição ao sol, aparece como uma grande mácula pigmentada, mas pode imitar verrugas com uma aparência verrucosa e não pigmentada (Jones et al., 2020). Deve-se suspeitar de melanoma quando apresenta assimetria, bordas irregulares, cor diversificada, diâmetro maior que $6 \mathrm{~mm}$, mudança recente incluindo tamanho, cor e forma e 
inflamação (Linares, Zakaria, \& Nizran, 2015).

Os fatores de risco gerais para o câncer de pele incluem a genética, como ter pele e olhos claros, cabelo loiro ou ruivo, nevos displásicos ou muitas manchas comuns, pele que queima, fica com sardas, vermelha ou dolorida após a exposição ao sol, além de diagnóstico prévio ou história familiar de câncer de pele e variantes genéticas ou mutações; e exposição aos raios ultravioleta, que pode induzir danos à pele e DNA (Watson, Holman, \& Maguire-Eisen, 2016). A radiação UV é o principal fator ambiental que afeta a função e a sobrevivência de muitos tipos de células e é considerada a maior causa na indução dos tumores cutâneos tipo basocelulares e espinocelulares (LaBerge et al., 2020). Radioterapia, exposição crônica ao arsênio, imunossupressão de longo prazo, infecção por vírus da imunodeficiência humana (HIV)/AIDS, infecção por papilomavírus e ceratose actínica também são citados na literatura (Gandhi \& Kampp, 2015).

O diagnóstico de câncer de pele é feito visualmente, com avaliação dermatoscópica da lesão, seguida de biópsia e avaliação histopatológica. Recentemente, muitas tecnologias inovadoras de detecção de câncer de pele foram desenvolvidas para aumentar a precisão do diagnóstico, como a microscopia confocal de refletância eletrônica, tomografia de coerência óptica, ultrassom de alta frequência, análise digital multiespectral de lesões cutâneas, espectroscopia de impedância elétrica, espectroscopia Raman, tomografia multifotônica e ensaio de lesão cutânea pigmentada (Dorrell \& Strowd, 2019). No entanto, dermatoscopia ainda é o melhor método para avaliar lesões cutâneas pigmentadas e não pigmentadas porque é preciso, rápido, de fácil manuseio e amplamente disponível. Estudos mostraram que, em comparação com o olho nu, a dermatoscopia aumenta a sensibilidade diagnóstica do melanoma em até 25\%, dependendo da experiência do médico (Weber et al., 2018).

O número de novos casos de câncer de pele tem aumentado desde 1970 devido, principalmente, às mudanças nos hábitos de lazer associadas à uma maior exposição aos raios ultravioleta. Com isso, salienta-se a importância da prevenção primária, que consiste na redução do comportamento de risco em relação ao câncer de pele (Kornek \& Augustin, 2013). Algumas recomendações feitas com esse propósito incluem usar chapéus, camisas de manga longa, óculos escuros e filtros solares com FPS acima de 30 e reaplicação adequada, ficar na sombra entre 10 e 16 horas e usar barracas de algodão ou lona em praias e piscinas (Sociedade Brasileira de Dermatologia, 2020). Já a prevenção secundária é constituída pelo rastreamento do câncer de pele (Federman, Kirsner, \& Viola, 2013). Nesse processo, os pilares são os testes de triagem e/ou exames, que permitem a detecção da doença em indivíduos assintomáticos (Lopez, Carvajal, \& Geskin, 2018).

Com base nisso, anualmente, a Sociedade Brasileira de Dermatologia (SBD) promove a Campanha Nacional de Prevenção ao Câncer da Pele (CNPCP), onde médicos dermatologistas fazem orientações sobre a exposição aos raios UV e exames gratuitos na população, em postos de saúde de grande parte do país, no horário das 9h às 15h (Sociedade Brasileira de Dermatologia, 2006).

Nesse âmbito, o objetivo deste estudo é avaliar os efeitos da Campanha Nacional de Prevenção ao Câncer de Pele, na cidade de Alfenas-Minas Gerais, nos anos de 2014 a 2018.

\section{Metodologia}

O presente estudo foi elaborado por meio de uma pesquisa documental que, de acordo com Junior (2021, p. 42), "é aquela em que os dados logrados são absolutamente provenientes de documentos, com o propósito de obter informações neles contidos, a fim de compreender um fenômeno". Segundo Gil (2002, p. 46), "nesta categoria estão os documentos conservados em arquivos de órgãos públicos e instituições privadas, tais como associações científicas [...]".

As informações obtidas durante a Campanha Nacional de Prevenção ao Câncer de Pele são compiladas e disponibilizadas na

forma de relatórios pela Sociedade Brasileira de Dermatologia por meio do sítio eletrônico <https://www.sbd.org.br/dezembroLaranja/estatisticas/>. 
Em agosto de 2020, foi realizado o acesso ao sítio eletrônico para obtenção dos relatórios da Campanha no município de Alfenas - Minas Gerais, entre 2014 e 2018, sobre os quais foi realizada uma pesquisa documental de caráter quantitativo. Os gráficos produzidos e análises realizadas através da pesquisa encontram-se nos resultados deste trabalho.

Na discussão, as análises são contextualizadas com literatura recente e fiável dos temas apontados. A conclusão dos autores e propostas para futuros trabalhos compõem as considerações finais.

\section{Resultados}

No Dia Nacional de Combate ao Câncer da Pele dos anos de 2014 a 2018, na cidade de Alfenas-MG, realizou-se um total de 1291 exames dermatológicos, sendo demonstrados, na Figura 1, os números de exames realizados em cada ano.

Figura 1 - Gráfico de pessoas examinadas no dia do Combate Nacional de Câncer de Pele de 2004 a 2018 na Cidade de Alfenas/MG.

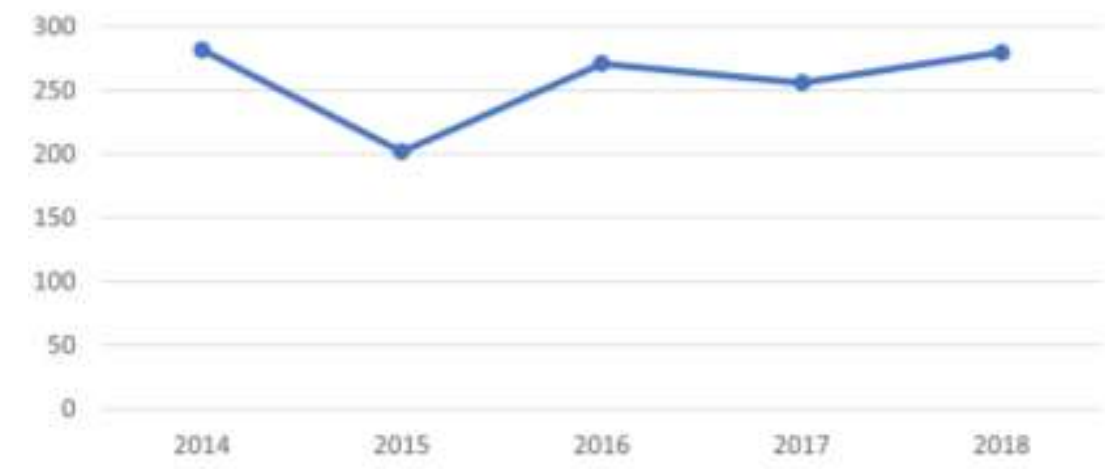

Fonte: Autores.

Na Figura 1, podemos observar o número de pessoas examinadas no dia da campanha em cada ano e como esse número se manteve estável ao longo dos anos, com queda no ano de 2015.

Foram diagnosticados 108 casos de câncer de pele ao todo, durante os anos de 2014 a 2018. E na Figura 2, podemos ver como foi a evolução dos diagnósticos de câncer de pele em cada ano da campanha.

Figura 2 - Gráfico de diagnósticos realizados no dia do Combate Nacional de Câncer de Pele de 2004 a 2018 na Cidade de Alfenas/MG.

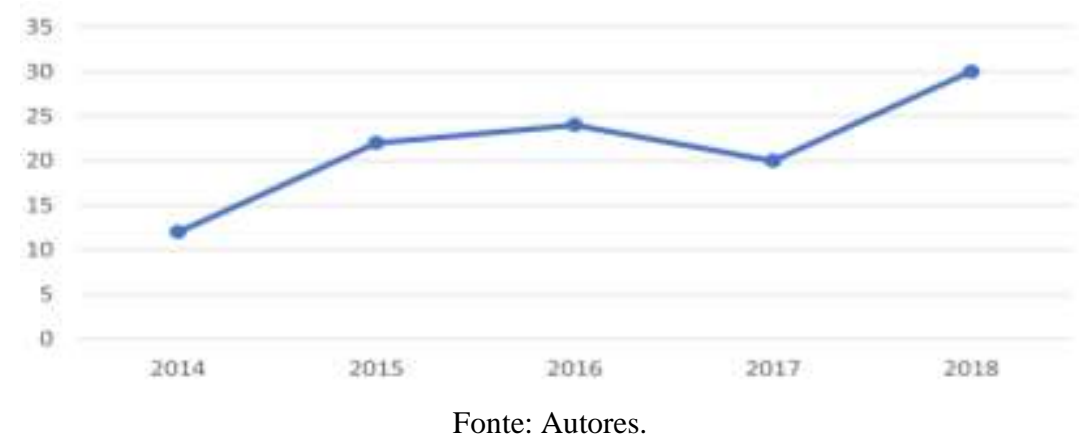

De acordo com a Figura 2, houve uma tendência de aumento no número de casos diagnosticados a cada ano, com uma leve queda em 2017 e novo aumento em 2018. 
Os meios de divulgação que motivaram as pessoas a participar da campanha foram amigos/família, cartaz/panfleto, palestras, $\mathrm{TV}$, rádio, jornal e outros, sendo este último, citado por $40 \%$ dos examinados.

Apesar da divulgação da campanha, muitas pessoas continuam se expondo ao sol sem proteção. A Figura 3 mostra a porcentagem de pessoas que se expõem ao sol, com ou sem proteção e de pessoas que não se expõem, nos anos de 2014 a 2018.

Figura 3 - Gráfico da condição de exposição ao sol dos examinados no dia do Combate Nacional de Câncer de Pele de 2004 a 2018 na Cidade de Alfenas/MG.

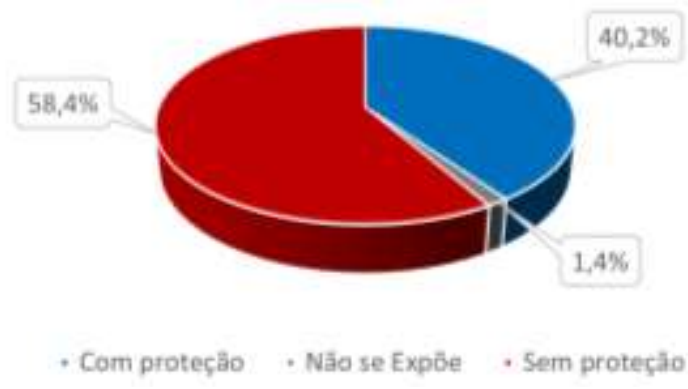

Fonte: Autores.

O número de pessoas que referiram exposição sem proteção foi maior em todos os anos, representando cerca de $58,4 \%$, comparado ao número dos que afirmaram exposição com proteção, 40,2 \%, como mostra a figura 3.

A figura 4 mostra a porcentagem de examinados do sexo masculino e do sexo feminino no dia da campanha, durante os anos de 2014 a 2018.

Figura 4 - Gráfico do sexo dos examinados no dia do Combate Nacional de Câncer de Pele de 2004 a 2018 na Cidade de Alfenas/MG.

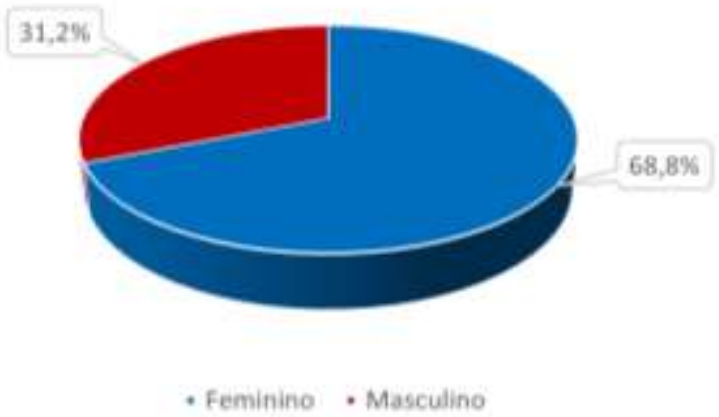

Fonte: Autores.

Conforme a Figura 4, houve predominância das mulheres nos exames dermatológicos realizados na campanha ao longo dos 5 anos, representando uma porcentagem de, aproximadamente, 68,8\%, em detrimento dos homens, 31,2\% aproximadamente.

Quanto ao risco de câncer de pele, um total de 756 examinados possuíam risco de desenvolver a doença e a Figura 5, mostra como foi essa evolução ao longo dos anos. 
Figura 5 - Gráfico do risco de câncer de pele em examinados no dia do Combate Nacional de Câncer de Pele de 2004 a 2018 na Cidade de Alfenas/MG.

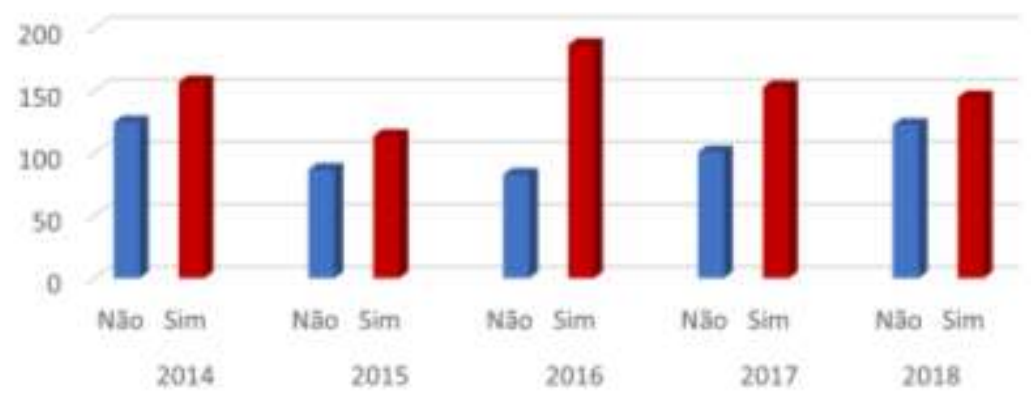

Fonte: Autores.

O número de pessoas com risco de desenvolver câncer de pele manteve-se maior durante todos os anos analisados (2014-2018) na campanha, em relação ao número de pessoas sem risco de desenvolver câncer de pele, como demonstra a Figura 5.

Ainda nesse público, foi possível analisar o número de pessoas que usavam fotoproteção ao se expor ao sol, em cada ano da campanha, conforme a Figura 6.

Figura 6 - Gráfico do uso de fotoproteção dos examinados no dia do Combate Nacional de Câncer de Pele de 2004 a 2018 na Cidade de Alfenas/MG.

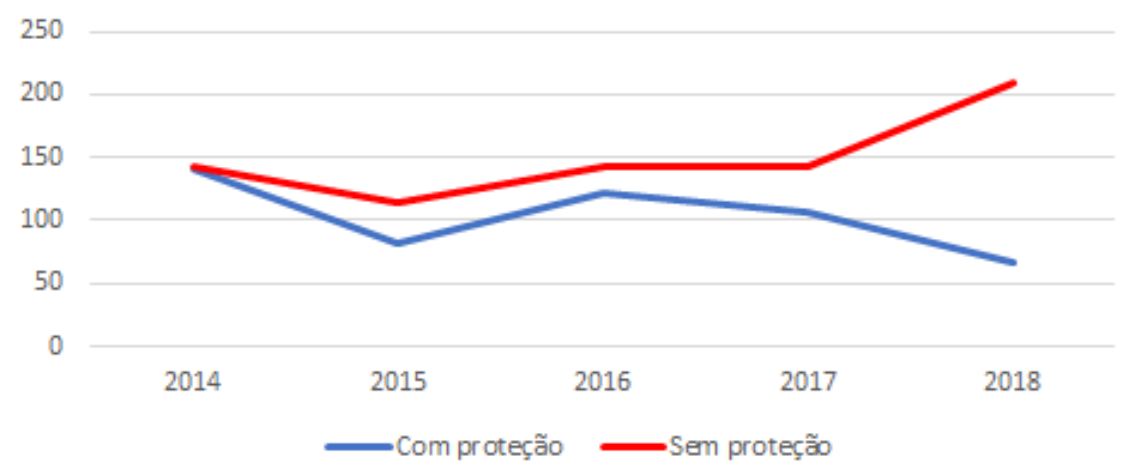

Fonte: Autores.

Na Figura 6, observa-se que o número de pessoas que utilizavam fotoproteção se manteve estável ao longo dos anos, entretanto entre 2017 e 2018, houve um grande aumento no número de pessoas que relataram se expor ao sol sem proteção, comparando-se aos anos anteriores.

\section{Discussão}

O câncer de pele é muito comum no Brasil, mas quando ele é descoberto no início, há mais de $90 \%$ de chances de cura (Sociedade Brasileira de Dermatologia, 2020). Com esse objetivo, os programas de rastreamento, como a Campanha Nacional de Prevenção ao Câncer da Pele, realizam atividades que permitem o diagnóstico precoce da doença, sendo possível reconhecer até $90 \%$ 
dos casos de melanoma em fases iniciais, especialmente se realizados em populações com fatores de risco presentes (Rocha et. al, 2002).

Nesse contexto, o diagnóstico precoce de cânceres não melanoma diminui as deformidades e sequelas cirúrgicas inestéticas, enquanto o de melanoma reduz a letalidade (Sociedade Brasileira de Dermatologia, 2006). Dessa forma, a Campanha Nacional de Prevenção ao Câncer da Pele (CNPCP), trouxe resultados promissores, sobretudo para o diagnóstico precoce do câncer de pele, desde que foi implantada no Brasil. Em Alfenas, de acordo com os resultados do período de 2014 a 2018 apresentados e vistos nas figuras 1 e 2, muitas pessoas tiveram a chance de serem examinadas e diagnosticadas devido à Campanha.

Pode-se correlacionar o aumento do número de casos diagnosticados com a maior divulgação da campanha, notadamente pelas mídias sociais, que vêm ganhando um maior destaque entre os meios de comunicação, o que permitiu que mais pessoas obtivessem informações a respeito do câncer de pele, despertando um maior interesse pelo auto-cuidado e, assim, tivessem acesso aos exames realizados pela campanha promovida pela SBD.

Esse tipo de câncer possui uma etiologia multifatorial, que combina fatores não modificáveis, como a genética, e modificáveis, como o ambiente (Watson, Holman, \& Maguire-Eisen, 2016). Contudo, o principal fator de risco é a exposição à radiação ultravioleta (UV), visto que a radiação ultravioleta agride diretamente o DNA celular, por meio de alterações estruturais e de oxidação (Júnior et al., 2020). Pela classificação da Fototipagem da pele de Fitzpatrick, os fototipos I e II são mais vulneráveis a esses danos, pois apresentam menor quantidade de melanina, a qual protege a epiderme de se queimar com mais facilidade. Além disso, maiores quantidade de nevos, predispõem o indivíduo ao maior risco de apresentar melanoma, assim como ter um diagnóstico prévio ou histórico familiar de câncer de pele, especialmente o melanoma (Watson, Holman, \& Maguire-Eisen, 2016).

Os brancos não hispânicos têm aproximadamente 25 vezes mais chances de apresentar melanoma em comparação com os negros e, aproximadamente, seis vezes em comparação aos hispânicos. Entretanto, a população negra e hispânica tem um pior prognóstico da doença, porque o diagnóstico normalmente é tardio.O CBC e CEC se associam a exposição cumulativa crônica, CBC nodular normalmente surge na região da cabeça, enquanto o $\mathrm{CBC}$ superficial, no tronco. Já o melanoma se associa à exposição intermitente e histórico de queimaduras solares (Watson, Holman, \& Maguire-Eisen, 2016). Pessoas que apresentam fatores de risco identificáveis procuram com mais frequência o dermatologista e ações como a da CNPCP, mas mesmo quem não apresenta esses fatores de risco, também podem procurar a CNPCP, porém em menor quantidade, como mostra a figura 5.

Apesar da Campanha buscar atingir toda a população, houve um predomínio de mulheres em todos os anos, como visto na figura 4. Essa discrepância entre os sexos dos indivíduos examinados demonstra uma maior preocupação feminina tanto em relação à estética da pele, quanto à prevenção de doenças, inclusive relativas à pele, sendo constatada pela predominância na utilização do sistema de saúde. Nesse sentido, também é possível relacionar uma maior tendência à exposição solar pelo sexo masculino com o tipo de atividade ocupacional realizada, como as profissões exercidas ao ar livre, que resultam em períodos prolongados de exposição ao sol, deixando esses indivíduos mais vulneráveis ao câncer de pele (Clavico et al., 2015). Um estudo de pesquisa de campo sobre o câncer de pele em trabalhadores rurais do município de Horizontina-RS revelou que, embora a maioria dos entrevistados soubessem do risco de câncer de pele, muitos ainda se expunham de maneira inadequada, permanecendo ao sol por mais de 3 horas. Soma-se, ainda, o agravante de que, essa exposição pode durar até o dia inteiro, em períodos de safra (Dalcin et.al., 2021).

O público presente na campanha possui acesso às informações sobre as atividades realizadas, data e hora dos atendimentos, assim como ao contato com o médico dermatologista, sendo que sua participação indica a preocupação com a saúde e estética da pele (Sociedade Brasileira de Dermatologia, 2006). Sendo assim, seria esperado que essa parcela da sociedade se prevenisse melhor em relação à exposição solar do que a população em geral. Todavia, apesar do câncer de pele ser o mais incidente no Brasil, com cerca de 180 mil novos casos ao ano, segundo dados do site da Sociedade Brasileira de Dermatologia (SBD), a porcentagem de indivíduos que se expõe sem proteção tem sido elevada e relativamente estável durante os anos analisados, com aumento em 2018, de acordo com as figuras 3 e 6. Nesse âmbito, um estudo transversal sobre o conhecimento populacional sobre prevenção e reconhecimento de sinais do 
câncer de pele, realizado por meio da aplicação de um questionário para 458 entrevistados, mostrou dados preocupantes, em que 33,84\% $(n=155)$ da amostra populacional relataram aplicar o protetor solar apenas quando se lembram, 27,73\% (n=127) negam o uso e 25,33\% ( $\mathrm{n}=116)$ aplicam somente vez ao dia. Além disso, 64,85\%, (n=297), responderam que não há a possibilidade de se exporem ao sol, sem risco à saúde, em horários de elevada radiação não fazendo o uso do protetor solar (Martins, Ivantes, \& Rocha-Brito, 2021). Isso pode indicar a necessidade de ampliar as ações educativas durante a campanha, a fim de não priorizar apenas o diagnóstico precoce, mas também informar sobre o câncer de pele, seus fatores de risco e prevenção.

Eventos como esse da CNPCP são de extrema importância, pois permitem o acesso direto do paciente ao médico dermatologista, visto que, em muitas regiões do Brasil, há falta desses profissionais no SUS ou frequentes problemas como a demora para agendar consultas, o que prejudica o diagnóstico precoce e consequentemente a redução da letalidade. E a Campanha Nacional de Prevenção ao Câncer da Pele leva a possibilidade de diagnóstico precoce a todas as classes sociais, por estar incluída na saúde pública.

\section{Considerações Finais}

A Campanha Nacional de Prevenção ao Câncer da Pele foi importante para examinar e diagnosticar pacientes durante o período analisado na cidade de Alfenas, mas seus dados também evidenciaram que os cuidados com a exposição solar pela população ainda precisam melhorar. Nesse âmbito, ainda é necessário investir na conscientização da população a respeito da prevenção primária ao câncer de pele, além de ampliar e divulgar, cada vez mais, a Campanha Nacional de Câncer de Pele, para que se atinja uma boa prevenção secundária, sobretudo para o público masculino, o qual participou menos das campanhas já realizadas. Desse modo, mais pessoas poderiam se prevenir e ter acesso a um diagnóstico precoce.

Ademais, são necessários novos estudos que tenham como objetivo relacionar a eficácia da CNPCP em outras cidades e estados brasileiros e, até mesmo, em nível nacional, utilizando os resultados encontrados para a criação de estratégias que otimizem sua efetividade.

\section{Referências}

Brandt, M. G., \& Moore, C. C. (2019). Nonmelanoma skin cancer. Facial Plastic Surgery Clinics, 27(1), 1-13.

Clavico, L. S., Trindade, G. S., Rodrigues, O., \& Trindade, R. A. R. (2015). Campanha de prevenção ao câncer da pele (Rio Grande-RS): perfil epidemiológico dos atendidos. Saúde e Pesquisa, 8(1), 113-123.

Dalcin, M. M., Krause , G. C. ., Scherer, C. M. ., Ceolin, S., Lautenschleger , G., \& Badke, M. R. . (2021). Skin cancer in rural workers: photoexposure and guindance on risk factors. Research, Society and Development, 10(1), e15110111594.

Dorrell, D. N., \& Strowd, L. C. (2019). Skin cancer detection technology. Dermatologic clinics, 37(4), 527-536.

Federman, D. G., Kirsner, R. S., \& Viola, K. V. (2013). Skin cancer screening and primary prevention: facts and controversies. Clinics in dermatology, 31(6), 666-670. Gil, A. C. (2002). Como elaborar projetos de pesquisa (Vol. 4, p. 175). São Paulo: Atlas.

Gandhi, S. A., \& Kampp, J. (2015). Skin Cancer Epidemiology, Detection, and Management. The Medical Clinics of North America, 99(6), $1323-1335$.

Instituto Nacional de Câncer José Alencar Gomes da Silva (2019). Estimativa 2020: incidência de câncer no Brasil.

Jones, O. T., Ranmuthu, C. K., Hall, P. N., Funston, G., \& Walter, F. M. (2020). Recognising skin cancer in primary care. Advances in therapy, 37(1), 603-616.

Junior, E. B. L., de Oliveira, G. S., dos Santos, A. C. O., \& Schnekenberg, G. F. (2021). Análise documental como percurso metodológico na pesquisa qualitativa. Cadernos da FUCAMP, 20(44).

Júnior, J. P. R., da Penha Pacheco, A., de Queiroz Turíbio, D. D. C., \& Jabour, T. B. F. (2020). Ação contra o câncer de pele em cidade com alto índice ultravioleta. Revista Brasileira em Promoção da Saúde, 33.

Kornek, T., \& Augustin, M. (2013). Skin cancer prevention. JDDG: Journal der Deutschen Dermatologischen Gesellschaft, 11(4), $283-298$.

LaBerge, G. S., Duvall, E., Grasmick, Z., Haedicke, K., Galan, A., Leverett, J., ... \& Pawelek, J. (2020). Focus: Skin: recent advances in studies of skin color and skin cancer. The Yale journal of biology and medicine, 93(1), 69. 
Research, Society and Development, v. 11, n. 1, e11811124237, 2021

(CC BY 4.0) | ISSN 2525-3409 | DOI: http://dx.doi.org/10.33448/rsd-v11i1.24237

Leiter, U., Keim, U., \& Garbe, C. (2020). Epidemiology of skin cancer: update 2019. In Sunlight, Vitamin D and Skin Cancer (pp. 123-139). Springer, Cham.

Linares, M. A., Zakaria, A., \& Nizran, P. (2015). Skin cancer. Primary care, 42(4), 645-659.

Lopez, A. T., Carvajal, R. D., \& Geskin, L. (2018). Secondary Prevention Strategies for Nonmelanoma Skin Cancer. Oncology (08909091), 32(4).

Martins, M. B., Ivantes, A. F. C., \& Rocha-Brito, K. J. P. (2021). Population knowledge about prevention and recognition of signs of skin cancer: A cross-section study. Research, Society and Development, 10(5), e36210515038.

Peris, K., Fargnoli, M. C., Garbe, C., Kaufmann, R., Bastholt, L., Seguin, N. B., Bataille, V., Marmol, V. D., Dummer, R., Harwood, C. A., Hauschild, A., Höller, C.

Haedersdal, M., Malvehy, J., Middleton, M. R., Morton, C. A., Nagore, E., Stratigos, A. J., Szeimies, R. M., Tagliaferri, L., ... European Dermatology Forum (EDF).

the European Association of Dermato-Oncology (EADO) and the European Organization for Research and Treatment of Cancer (EORTC) (2019). Diagnosis and treatment of basal cell carcinoma: European consensus-based interdisciplinary guidelines. European journal of cancer, 118, $10-34$.

Rocha, F. P. D., Menezes, A., Almeida Junior, H. L. D., \& Tomasi, E. (2002). Especificidade e sensibilidade de rastreamento para lesões cutâneas pré-malignas e malignas. Revista de Saúde Pública, 36, 101-106.

saes da Silva, E., Tavares, R., da Silva Paulitsch, F., \& Zhang, L. (2018). Use of sunscreen and risk of melanoma and non-melanoma skin cancer: a systematic review and meta-analysis. European Journal of Dermatology, 28(2), 186-201.

Sociedade Brasileira de Dermatologia (2006). Análise de dados das campanhas de prevenção ao câncer da pele promovidas pela Sociedade Brasileira de Dermatologia de 1999 a 2005. Anais brasileiros de dermatologia, 81(6), 533-539.

Sociedade Brasileira de Dermatologia. Câncer da pele. https://www.sbd.org.br/dermatologia/pele/doencas-e-problemas/cancer-da-pele/64/

Watson, M., Holman, D. M., \& Maguire-Eisen, M. (2016, August). Ultraviolet radiation exposure and its impact on skin cancer risk. In Seminars in oncology nursing (Vol. 32, No. 3, pp. 241-254). WB Saunders.

Weber, P., Tschandl, P., Sinz, C., \& Kittler, H. (2018). Dermatoscopy of neoplastic skin lesions: recent advances, updates, and revisions. Current treatment options in oncology, 19(11), 1-17. 